\title{
Mechanical equilibria in inclusion-host systems
}

Zhang, Youxue

Department of Geological Sciences, University of Michigan, Ann Arbor, MI 48109-1063

Mineral, melt/glass, and fluid inclusions in a host mineral are common and have been used widely to infer mantle processes, source conditions, and P-T history. When an inclusion and its host mineral form at some initial temperature and pressure, the inclusion pressure is presumably the same as the confining pressure acting upon the host. Subsequently when the $\mathrm{P}-\mathrm{T}$ conditions change, the pressure inside the inclusion is expected to be different from that on the host if compressibilities and thermal expansivities of the inclusion and host are different. Several pioneers have studied the mechanical and phase equilibria of inclusionhost systems (e.g., Rosenfeld and Chase, 1961; Harris and Milledge, 1970; Adams et al., 1975; Graham and Cybriwsky, 1981; Gillet et al., 1984; Tait, 1992).

The P-T history for an inclusion-host pair of mantle origin is often complicated, including incorporation of the inclusion at presumably equilibrium conditions, then slow P-T variation in the mantle, then rapid decompression when the sample is brought up to the surface by an eruption, and then rapid cooling to surface temperature. When trying to explain features related to inclusion-host systems, it is important to bear in mind the complexity of the P-T history and to understand which part of the P-T history caused a given feature. Chemical reactions (especially those that involve diffusion) and viscous relaxation can only happen at high temperatures and when there is enough time. On the other hand, elastic deformation, plastic yielding, and fracturing can happen rapidly regardless of the temperature. In this presentation, I investigate mechanical and phase equilibria in inclusion-host systems by adapting textbook solutions (Love, 1944; Hill,1951; Timoshenko and Goodier, 1970) to geological problems. For an inclusion-host system that is initially under lithostatic pressure and in mechanical and thermodynamic equilibria, the new elastic and plastic equilibrium and viscous relaxation are considered.

If the inclusion and host are concentric spheres and elastically isotropic, as T-P conditions on the inclusion-host pair change (assuming that the pressure on the outer surface of the host is isotropic), the host becomes stressed and the inclusion is under isotropic stress but the inclusion pressure is typically different from that on the outside surface of the host. The relative change of the inclusion volume also differs from that of the host; the difference is 0.75 times the pressure difference divided by the shear modulus of the host. Different inclusions in a single host may be under different pressures.

The assumption of elastic isotropy for the host mineral is important for simple solutions to be obtained and is often employed, but, to my knowledge, its accuracy has never been examined before. Recently, Schmidt et al. (1996) carried out experiments to examine the variation of volume and pressure of an $\mathrm{H}_{2} \mathrm{O}$ fluid inclusion in quartz as a function of T and P [I.-M. Chou, personal communications]. The pressure inside the inclusion is determined by observing the complete disappearance of the vapor phase in a vapor-liquid inclusion by varying the temperature at a given host pressure. The pressure in the inclusion is then the saturation pressure at the given temperature and molar volume at the P-T can be calculated. They found that when the inclusion volume changed by $-1.9 \%$ relative, the calculated volume change for the host quartz crystal is only $-0.7 \%$ considering only the compression and thermal expansion of quartz. Including the effect of elasticity but assuming elastic isotropy for quartz, the calculated inclusion volume variation is $-1.2 \%$. The agreement with the observed $-1.9 \%$ variation is better, but still not good, suggesting that elastic anisotropy of 
quartz may have to be considered. Love (1944) gave a solution for a spherical inclusion in an elastically anisotropic solid with transverse isotropy about the radius vector. Even though quartz does not even possess transverse isotropy, this solution is expected to approximate the elastic properties of quartz better than perfect elastic isotropy. Incorporating the anisotropy, the calculated inclusion volume variation is $-1.8 \%$, in good agreement with the observed $2.0 \%$. Therefore, for accurate treatment of the elastic effects involving elastically anisotropic host, anisotropy must be incorporated. Because solutions for elastically anisotropic materials are few and complex, the following discussion still assumes elastic isotropy.

When the inclusion pressure is not the same as the pressure on the outside surface of the host, Tait (1992) solved for the viscous flow velocity near the inclusion-host boundary by assuming (i) that the host is an incompressible Newtonian fluid that flows radially, and (ii) that the host is much more viscous than the inclusion. Using this velocity, he assumed that the characteristic time scale for viscous relaxation is the inclusion radius divided by the velocity, and obtained the time scale for stress relaxation in the host-inclusion system. This relaxation time scale is in error because particles in the host phase around the inclusion do not have to flow a distance of the inclusion radius to release the stress, instead, particles only have to move such a distance that the volume of the inclusion changes from that corresponding to inclusion pressure to that corresponding to the outside pressure. In this way, the relaxation time scale for the inclusion-host system with a constant inclusion bulk modulus can be found to be 1.33 times the host viscosity divided by the inclusion bulk modulus. Compared with the viscous relaxation time scale of the host phase without the presence of inclusions, the Maxwell relaxation time scale, the relaxation time scale for the inclusion-host system differs by a factor of 1.33 times the host shear modulus divided by inclusion bulk modulus. Using this result, for a typical upper mantle mineral olivine with a viscosity of $1 \mathrm{E} 21 \mathrm{~Pa}$ and a typical solid inclusion bulk modulus of $1 \mathrm{E} 11 \mathrm{~Pa}$, the relaxation time scale is about 400 years, a short time geologically. Hence the stress in an olivine crystal that contains inclusions is expected to be lithostatic in much of the upper mantle with normal temperatures. However, if a mantle xenolith is brought up rapidly by volcanic eruption, there would not be enough time for stress relaxation and there will be anisotropic stress in an inclusion-bearing olivine crystal due to rapid decompression. This stress field should not be interpreted to be that in olivine in the mantle. Mineral viscosity increases rapidly with decreasing temperature. For example, Karato (1997) estimated that at $973 \mathrm{~K}$, mantle mineral viscosity is $1 \mathrm{E} 32 \mathrm{~Pa}$ s. At such a viscosity, the stress relaxation time scale would be 4 orders of magnitude greater than the age of the earth. Hence under such low temperatures, anisotropic stress would be common if there are inclusions in a mineral. For diamond and garnet, the viscosity is not well-known but presumably very high. Hence viscous relaxation in these minerals is often negligible.

Although stress distribution in the host in the presence of plastic yielding may be calculated, plastic yielding may significantly complicate interpretation of the stress history (Harris and Milledge, 1970; Graham and Cybriwsky, 1981). Fractures develop when the stress intensity factor or the crack extension force exceeds a critical value. Radiating fractures are often observed around inclusions in garnet megacrysts of mantle origin only when the inclusion bulk modulus is smaller than the host bulk modulus. They are inferred to have formed during rapid decompression and can be used to constrain decompression history.

Inclusions in minerals have often been used to infer temperature and pressure. If $\mathrm{T}$ is greater than $1300 \mathrm{~K}$, viscous relaxation is rapid for normal mantle minerals (such as olivine). Hence inclusion-host pairs can be used in the usual way to infer the last equilibrium T-P. However, If $\mathrm{T}$ is smaller than $1000 \mathrm{~K}$, or more rigid mantle minerals (such as diamond and garnet) are considered, viscous relaxation ma be negligible and use of inclusion-host pairs for 
thermobarometry requires caution. A valid application is to use the inclusions to infer or constrain the formation conditions (instead of peak $T$ after formation) if there is no reaction between the host and the inclusions or if the reactions are so slow that original inclusion compositions are preserved in their cores. If a single inclusion contains several phases, the equilibria between the several phases can be used to infer $\mathrm{T}$ (peak temperature or closure temperature) and inclusion pressure. However, inclusion pressure may not have a depth significance. If the host and the inclusion can react, the observed compositions of the inclusion and host reflect the long P-T history experienced by the pair and cannot be simply used to infer formation P-T or the peak P-T. Hunter and Smith (1981) and Smith and Barron (1991) used the Fe-Mg exchange equilibrium between orthopyroxene inclusion and garnet host and the $\mathrm{Al}$ content in orthopyroxene inclusion in garnet to infer T-P conditions or paths for the inclusion-host pairs. However, because the estimated temperatures are low (770 to $1070 \mathrm{~K}$ ), viscous relaxation in the garnet host is expected to be negligible. The estimated pressures are inclusion pressures that cannot be used to estimate depth (and hence cannot be used to construct a geotherm). When there are several separate inclusions in a host mineral, the pressure in one inclusion (such as an orthopyroxene inclusion in a garnet) may not be the same as the pressure in another inclusion (such as a clinopyroxene inclusion in the same garnet) unless the host has viscously relaxed. Therefore, one may not combine several inclusions to solve for both peak $\mathrm{T}$ and $\mathrm{P}$. Correct "reading" of information stored by inclusion-host pairs requires an understanding of mechanical and phase equilibria involving the inclusion and host.

\section{References}

Adams, H. G., Cohen, L. H., and Rosenfeld, J. L., 1975, Solid inclusion piezothermometry I : Comparison dilatometry: Am. Mineral., v. 60, p. 574-583.

Gillet, P., Ingrin, J., and Chopin, C., 1984, Coesite in subducted continental crust: P-T history deduced from an elastic model: Earth Planet. Sci. Lett., v. 70, p. 426-436.

Graham, E. K., and Cybriwsky, Z. A., 1981, Garnet inclusions in diamond and the state of the upper mantle: Phys. Chem. Mineral., v. 7, p. 216-222.

Harris, J. W., Milledge, H. J., Barron, T. H. K., and Munn, R. W., 1970, Thermal expansion of garnets included in diamond: J. Geophys. Res., v. 75, p. 5775-5792.

Hill, R., 1950, The Mathematical Theory of Plasticity: Oxford, Oxford U. Press, 356 p.

Hunter, W. C., and Smith, D., 1981, Garnet peridotite from Colorado Plateau ultramafic diatremes: Hydrates, carbonates, and comparative geothermometry: Contrib. Mineral. Petrol., v. 76, p. 312-320.

Karato, S., 1997, Phase transformations and rheological properties of mantle minerals, in Crossley, D., and Soward, A. M., eds., Earth's Deep Interior: New York, Gordon and Breach, p. 223-272.

Love, A. E. H., 1944, Mathematical Theory of Elasticity: New York, Dover, 643 p.

Rosenfeld, J. L., and Chase, A. B., 1961, Pressure and temperature of crystallization from elastic effects around solid inclusions in minerals?: Am. J. Sci., v. 259, p. 519-541.

Schmidt, C., Chou, I.-M., Bodnar, R. J., and Bassett, W. A., 1996, Application of the hydrothermal diamond-anvil cell for microthermometric analysis of volatile-rich fluid inclusions: Eos, v. 77, p. F775.

Smith, D., and Barron, B. R., 1991, Pyroxene-garnet equilibration during cooling in the mantle: Am. Mineral., v. 76, p. 1950-1963.

Tait, S., 1992, Selective preservation of melt inclusions in igneous phenocrysts: Am. Mineral., v. 77, p. 146-155.

Timoshenko, S. P. and Goodier, J. N. 1970, Theory of Elasticity: New York, McGraw-Hill. 